\title{
The genus Eugenia L. (Myrtaceae) in India
}

\author{
Shareef S $\mathrm{M}^{*}$ \& E S Santhosh Kumar \\ Jawaharlal Nehru Tropical Botanic Garden \& Research Institute, Palode, Karimancode P.O., Thiruvananthapuram 695 562, Kerala, India \\ *Email: smshariftbgri@gmail.com
}

\section{ARTICLE HISTORY}

Received: 09 March 2020

Accepted: 09 April 2020

Published: 01 July 2020

KEYWORDS

Endemic; Eugenia; India; nomenclature; taxonomy

\section{ABSTRACT}

This paper provides a comprehensive taxonomic account of the genus Eugenia L. occurring in India. A total of 25 species and two varieties have been enumerated, of which 21 taxa are endemic to the Western Ghats. Eugenia macrosepala, an endemic species of the Western Ghats is reinstated here. Each species is provided with a short description, well known synonyms, types, distribution and phenology. An identification key is also provided.

\section{Introduction}

The genus Eugenia L., the second largest genus in Myrtaceae family, comprises about 1,100 species (1), which are mainly distributed in Brazil, Africa, SouthEast Asia and Malaysia (2, 3). Duthie (4) enumerated 131 species for erstwhile British India covering the geographical and political boundaries of India, Pakistan, Afghanistan, Nepal, Tibet, Myanmar, Bangladesh, Malayan peninsula and Sri Lanka. But he has followed a wider generic concept and accommodated Syzygium Gaertner in Eugenia.

Recent anatomical, palynological and molecular studies found that Eugenia and Syzygium are in quite different clades in which Eugenia was included in the Myrteae (usually in the subtribe Eugeniinae O. Berg) and Syzygium in the new Syzygiea clade (5-10). Based on the above concept the Indian Eugenia had easily differentiated from Syzygium by the young shoots and flowers often pubescent, flowers usually solitary or aggregated in many flowered axillary dichasial or raceme like inflorescences and seeds with fused cotyledons. Whereas in Syzygium, the young shoots and flowers are usually glabrous, the inflorescences usually terminal (sometimes rami- or cauliflorous) or paniculate with free cotyledons in the seeds.

After the tremendous contribution by Duthie (4), several state and provincial treatments have been completed in which notable contributions were made by several workers (11-30). In addition to these, many new taxa were also been published from different parts of the country (31-40). But national wide documentation of Indian Eugenia is far from complete. As part of the revisionary study on the genus Eugenia of India, the paper presents a synoptic account on Indian Eugenia with a brief description, synonyms, herbarium types, distribution, flowering and fruiting, identification key, etc. The present study reports a total of 25 species and two varieties from India and all are represented in the Western Ghats, of which 21 are strict endemics to this region.

\section{Key to the species}

1. Young Brachlets glabrous.............................................2

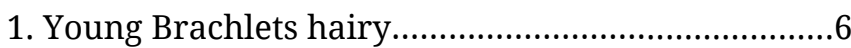

2. Leaves cordate at base.................... s. singampattiana

2. Leaves not cordate at base............................................

3. Leaves broadly elliptic to suborbicular...E. codyensis

3. Leaves otherwise......................................................

4. Fruits ribbed. E. uniflora

4. Fruits smooth. . .5

5. Sepals broadly ovate E. seithurensis

5. Sepals orbicular. E. calcadensis

6. Sepals ovate or broadly ovate.....................................

(c) Shareef \& Kumar (2020). This is an open-access article distributed under the terms of the Creative Commons Attribution License, which permits unrestricted use, distribution, and reproduction in any medium, provided the original author and source are credited (https://creativecommons.org/licenses/by/4.0/).

To cite this article: Shareef SM, Kumar ESS. The genus Eugenia L. (Myrtaceae) in India. Plant Science Today. 2020;7(3):360- 370. https://doi.org/10.14719/pst.2020.7.3.770 
6. Sepals not as above

7. Leaves silvery pubescent below. E. argentea

7. Leaves not silvery pubescent below .8

8. Flowers sessile to subsessile; fruits white, irregularly globose. E. memecylifolia

8. Flowers pedicellate; fruits not as above. . .9

9. Leaves spathulate or obovate. E. mabaeoides

9. Leaves neither spathulate nor obovate. .10

10. Bracteoles exceeding calyx lobes in length E. aloysii

10. Bracteoles always shorter than calyx lobes. .11

11. Leaves narrowly oblanceolate E. rottleriana

11. Leaves not as above. . .12

12. Flowers c. $2.5 \mathrm{~cm}$ across at anthesis; fruits globose .E. megamalayana

12. Flowers c. $1.2-1.5 \mathrm{~cm}$ cm across at anthesis; fruits subglobose. E. roxburghii

13. Sepals and petals orbicular or sub orbicular......14

13. Sepals and petals never orbicular or suborbicular ..17

14. Calyx lobes acute at apex

.E. terpnophylla var. keralensis

14. Calyx lobes obtuse at apex. .15

15. Pedicels $1.5-2.5 \mathrm{~cm}$ long. E. indica

15. Pedicels less than $1 \mathrm{~cm}$ long. . .16

16. Bracts lanceolate, petals orbicula E. discifera

16. Bracts oblong, petals obovate. E. codyensis var. obovata

17. Leaves obovate-oblanceolate, sepals oblong. E. velliangiriana

17. Leaves and sepals otherwise .18

18. Sepals deltoid; fruits oblongoid...E. anamalaiensis

18. Sepals and fruits not as above. .19

19. Flowers sessile, sepals orbicular....E. macrosepala

19. Flowers pedicellate, sepals not orbicular .20

20. Leaves and fruits densely floccose. E. floccosa

20. Leaves and fruits not floccose. .21

21. Flowers supra-axillary, $2-3 \mathrm{~cm}$ across at anthesis E. shettyana

21. Flowers not supra-axillary, always less than $2 \mathrm{~cm}$ across at anthesis.

22. Sepals lanceolate or suborbicular to ellipticoblong; ovule pendulous. 23

22. Sepals not as above, ovules in axile placentation .24

23. Young shoots fulvous tomentose; fruits hairy E. wynadensis

23. Young shoots silvery pubescent, fruits glabrous..
24. Petals oblong to narrowly elliptic-oblong...........25

24. Petals ovate-orbicular or orbicular. .26

25. Bracteoles lanceolate; sepals deltoid.

E. terpnophylla

25. Bracteoles oblong; sepals orbicular-ovate. E. mooniana

26. Young shoots grey pubescent; leaves elliptic-ovate, sepals ovate-orbicular E. agasthiyamalayana

26. Young shoots tawny silvery pubescent; leaves spathulate, sepals orbicular-ovate.

E. manickamiana

Eugenia agasthiyamalayana Gopalan \& Murugan, Indian J. Forest.31(4): 641. 2008.

Shrub to small trees, 2-8 m high; branchlets finely grey pubescent. Leaves elliptic-ovate, 2.5-6x 1.5-3 $\mathrm{cm}$, thin coriaceous, attenuate at base, caudateacuminate at apex; lateral nerves c. 12 pairs; petioles 0.7-1 cm long. Inflorescence supra-axillary or axillary (solitary or paired) or terminal 4-6 flowered racemes; peduncle c. $5 \mathrm{~mm}$ long. Bracteoles 2, linear, c. $2 \times 0.5 \mathrm{~mm}$. Flowers c. $1 \mathrm{~cm}$ across; pedicels slender, 5-12 mm long. Calyx tube c. $2 \mathrm{~mm}$ long; lobes 4, subequal, ovate to orbicular, $3-4.5 \mathrm{x} 2-2.5 \mathrm{~mm}$, fulvous pubescent without; petals 4 , orbicular, 4-4.5x 3-3.5 mm, white. Fruits globose, c. $2 \mathrm{~cm}$ across.

Type: INDIA, Tamil Nadu, Tirunelveli Distr., Agasthiyamalai Hills, Kallar to Sangumuthirai, 23 April 1992, c. 1400 m, R. Gopalan 99371 (CAL).

Distribution: Tamil Nadu; 1300-1800 m.

Flowering \& fruiting: January-October.

Eugenia aloysii C.J.Saldanha, Fl. Karnataka 2: 23. 1996.

Shrubs or small trees; branchlets fulvous tomentose. Leaves elliptic, 3.8 $-5.8 \times 1.1-2.4 \mathrm{~cm}$, rounded at base, acuminate at apex; petioles short. Flowers solitary or fascicled, axillary; pedicels 2-3 cm long, densely fulvous tomentose; bracteoles 2, linear, fulvous tomentose, exceeding calyx lobes. Calyx lobes 4, ovate, densely fulvous tomentose without; petals 4, white.

Type: INDIA, Karnataka, Hassan distr., near Genkalbetta, 19.12.1969, Saldahna 15931 (JCB).

Distribution: India (Karntaka); above c.1000 m.

Flowering: December.

Eugenia anamalaiensis E.S.S. Kumar, Veldk. \& Shareef, Webbia 69(1): 101. 2014. Eugenia gracilis Bedd, Madras J. Lit. Sci. III, 1: 46.1864 \& in Trans. Linn.Soc.25: 217. 1865 non E. gracilis O.Berg (1856). Eugenia mooniana Wight var. gracilis (Bedd.) Duthie in Hook. f., Fl. Brit. India 2: 505. 1879. Eugenia cotinifolia Jacq. var. gracilis (Duthie) M.R. Almeida, Fl. Maharashtra 2: 265. 1988. 

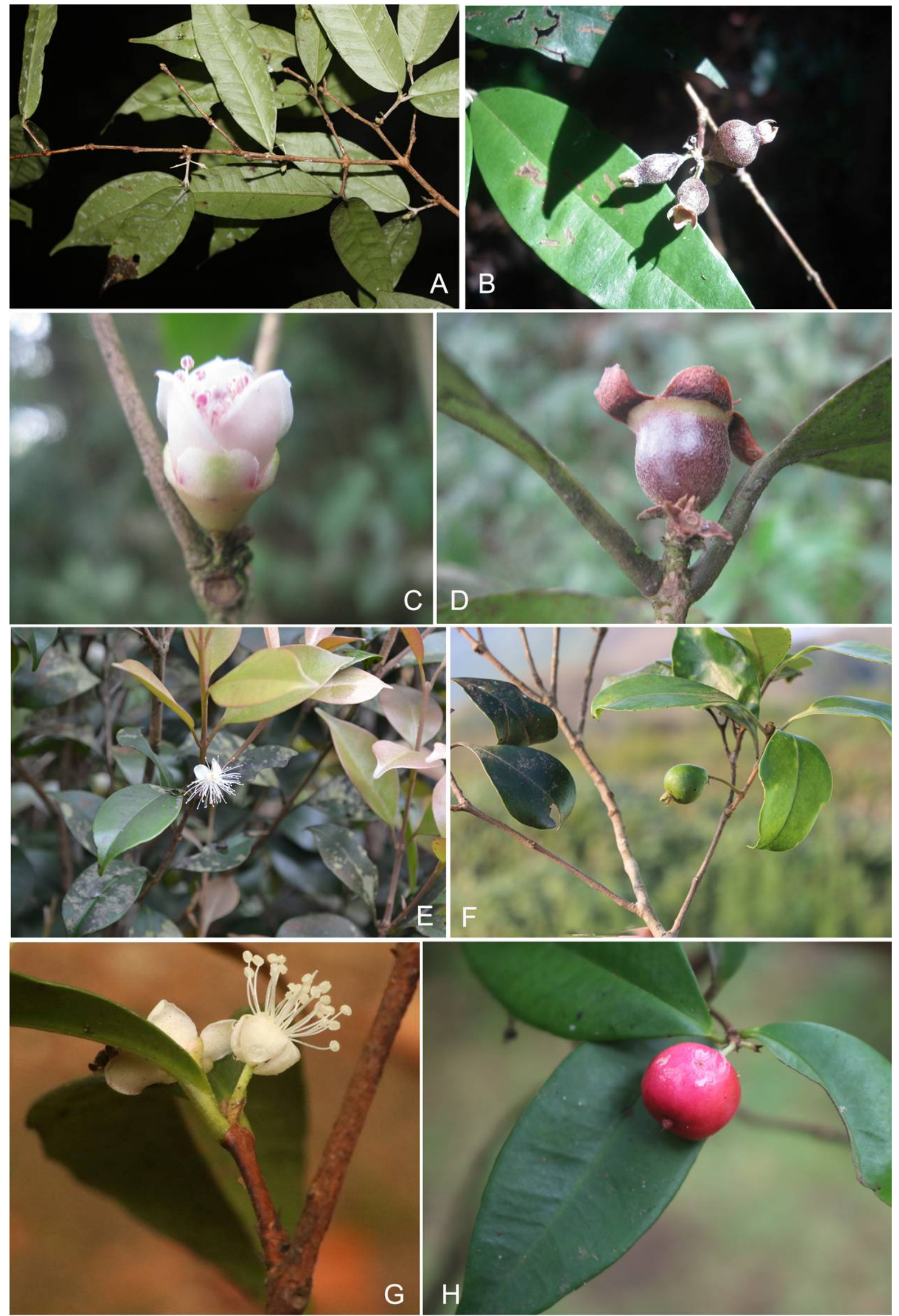

Fig. 1. A B. Eugenia argentea, C-D. E. codyensis, E-F. E. discifera, G-H. E. kalamii.

Large shrubs or small trees, to $6 \mathrm{~m}$; branchlets puberulous. Leaves lanceolate, $7.5-14 \mathrm{x} 2.3-4.5 \mathrm{~cm}$, attenuate to cuneate at base, acuminate to caudateacuminate at apex; lateral nerves c. 22 pairs; petiole
$0.5-0.8 \mathrm{~cm}$ long. Flowers white, axillary, supraaxillary rarely terminal or lateral; solitary or from short peduncles. Bracts 2. Calyx tube campanulate, 2-3.5 mm long; lobes 4, deltoid; petals 4, ovate- 
elliptic, white. Fruits oblong, rarely globose, to $2.5 \times$ $1.5 \mathrm{~cm}$, crimson on ripening; seeds 1-2.

Type: INDIA, Kerala, Anamalai Hills, R.H. Beddome, s.n. (BM).

Distribution: Maharashtra, Kerala; c.1350 m.

Flowering \& Fruiting: February-March (Maharashtra); October-February (Kerala).

Eugenia argentea Bedd., Fl. Sylv. S. India: 109. 1872. (Fig.1. A-B).

Shrub or small trees, to $5 \mathrm{~m}$; branchlets silverypubescent. Leaves lanceolate to elliptic-lanceolate, $10-13 \mathrm{x} 1.5-3 \mathrm{~cm}$, chartaceous, rounded at base, acuminate to caudate-acuminate at apex, silverypubescent beneath; lateral nerves 14-17 pairs; petiole 4-6 mm long. Flowers 1-1.2 cm across, creamy-white, solitary or fascicled in the leaf-axils or in very short cymes; pedicels 0.5-1.5 mm long. Bracteoles 2, linear lanceolate or filiform, c. $3.5 \mathrm{~mm}$ long, exceeding the calyx lobes. Calyx-tube nearly globose, c. $0.5 \mathrm{~cm}$ across; lobes 4 (rarely 5), ovate, pubescent without; petals 4 (rarely 5), suborbicular, $5 \times 4 \mathrm{~mm}$, white. Fruit depressed- globose or ellipsoid, 2.2x $1 \mathrm{~cm}$, dark yellow or brownish yellow, shortly appressedpubescent; seeds 1-4.

Type [Lectotype designated by Kumar et al. (41)]: INDIA, Kerala, Wayanadu ('Wynad') dist., Thamarassery Ghats ('Thambracherry Ghat'), Col. R.H. Beddome, s.n. (K) (Barcode K000518029).

Distribution: INDIA (Tamil Nadu \& Kerala), Endemic; 800-1500 m.

Flowering \& Fruiting: Throughout (Tamil Nadu \& Kerala).

Eugenia calcadensis Bedd., Fl. Sylv. S. India: 110. 1872.

Small trees, to $10 \mathrm{~m}$ high; young branchlets rusty pubescent. Leaves obovate-oblanceolate, rarely elliptic, 3.5-10x 1-4 cm, coriaceous, cuneate or attenuate at base, obtuse at apex; lateral nerves 6-10 pairs; petiole $0.8-1.2 \mathrm{~cm}$ long. Flowers c. $3 \mathrm{~cm}$ across, white, in axillary pairs or rarely solitary on short peduncle; pedicel 2-3 cm long. Bract linear-subulate, c. $5 \mathrm{~mm}$ long; bracteoles 2, c. $2 \mathrm{~mm}$ long. Calyx tube cup shaped, 3-5x 4-7 mm, greenish-white; lobes 4, orbicular, 4-6 mm diam, appressedly brown wooly tomentose without; petals 4 , oblong, $1.2-1.8 \mathrm{x} 0.8-1.4$ $\mathrm{cm}$. Fruit ellipsoid or globose, $0.8-1.5 \times 0.7-1 \mathrm{~cm}$, fulvous tomentose; seeds 1-2.

Type [Lectotype designated by Byng et al. (42)]: INDIA, Tamil Nadu, Calcad hills, South Tinnevelly, 2000ft., Beddome 2923 (BM) (Barcode BM0000944119).

Distribution: Tamil Nadu, Kerala; 200-1100 m.

Flowering \& Fruiting: June-November (Tamil Nadu); April-May (Kerala).

Eugenia codyensis Munro ex Wight, Ill. Ind. Bot. 2: 13. 1841. Eugenia cotinifolia subsp. codyensis (Munro ex Wight) P.S.Ashton in Revis. Handb. Fl. Ceylon 2: 412. 1981. Eugenia hypoleuca Thwaites ex Kosterm. in Quart. J. Taiwan Mus. 34: 164. 1981. Syzygium codyensis (Munro ex Wight) Chandrab. in Biol. Mem. A2: 57. 1977. (Fig. 1. C-D).

Small trees, to $6 \mathrm{~m}$ high; branchlets glabrous. Leaves broadly elliptic to suborbicular, 3-9x 2-4.5 cm, thickly coriaceous, broadly cuneate at base, retuse, obtuse, or subacute at apex; lateral nerves 10-12 pairs; petiole 5-10 mm long. Flowers white to pale yellow, $2 \mathrm{~cm}$ across, axillary solitary, rarely in fascicles, or rarely in terminal heads at end of abortive branchlets; pedicels $3.5 \mathrm{~cm}$ long. Bracteoles 2 , ovate, white tomentose. Calyx tube shallowly cupshaped, $8 \times 7 \mathrm{~mm}$, white pubescent; lobes 4, orbicular. Petals white, elliptic-spathulate, to $15 \times 10$ $\mathrm{mm}$. Fruit globose, $2.5 \mathrm{~cm}$ diam., ripening green flushed with crimson; seeds 1-2.

Type: INDIA, Karnataka, Coorg, near Mercara, Wight s.n. (K) (Barcode K000821511).

Distribution: India (Karnataka, Kerala, Maharashtra \& Tamil Nadu), Sri Lanka, Mauritius; above $900 \mathrm{~m}$.

Flowering \& Fruiting: March-May (Maharashtra); March-April (Karnataka); August-September (Tamil Nadu); January-August (Kerala).

Eugenia codyensis var. obovata Rijuraj, Rajendraprasad, Shareef \& Shaju, Ann. Plant Sci. 9(3): 3750. 2020.

Bushy shrub or small trees, to $3 \mathrm{~m}$ high; bark grayishwhite, rough; branchlets terete, younger shoots sparsely pubescent, glabrous when mature. Leaves narrowly elliptic to elliptic-oblong, 7-9x 2.5-3.5 cm, thinly coriaceous, punctate on both surfaces, attenuate at base and narrowly acuminate at apex, margin slightly revolute, nerves $10-14$ pairs, petioles 0.8-1 cm long. Flowers creamy-white, c. $1.2 \mathrm{~cm}$, usually 3-5 and rarely solitary, axillary and terminal, sessile or subsessile, pedicels to $0.5 \mathrm{~cm}$ long, golden pubescent; bracts 2, oblong, c. $1.5 \times 0.5 \mathrm{~mm}$, obtuse at apex, ciliate at the margin; bracteoles 2, ovate-deltoid, $1.5 \mathrm{x} 2 \mathrm{~mm}$, pubescent. Sepals 4, glandulate; lobes orbicular, rounded at apex, margin sparsely ciliate, persistent. Petals 4, obovate, $5 \times 3 \mathrm{~mm}$, free, creamywhite, pubescent along margins, glandulate, reflexed, caducous after anthesis. Fruit depressed globose, c. $0.8-0.9 \mathrm{x} 0.9-1.2 \mathrm{~cm}$, green, pubescent at base and apex, sparsely at the center, crowned with persistent calyx lobes. Seeds 2, rarely solitary, planoconvex.

Type: INDIA, Kerala, Kasaragod district, Ananthapura, $\pm 103 m$, January 2019. Shaju \& Rijuraj 93849 (TBGT).

Distribution: Kerala.

Flowering \& Fruiting: January-February.

Eugenia discifera Gamble, Bull. Misc. Inform. Kew 1918 (7): 239. 1918. (Fig. 1. E-F).

Small trees, to $5 \mathrm{~m}$ high; young branchlets rusty pubescent. Leaves elliptic-ovate or obovate, 3-5x 1.5-3 cm, coriaceous, attenuate at base, obtuse or 


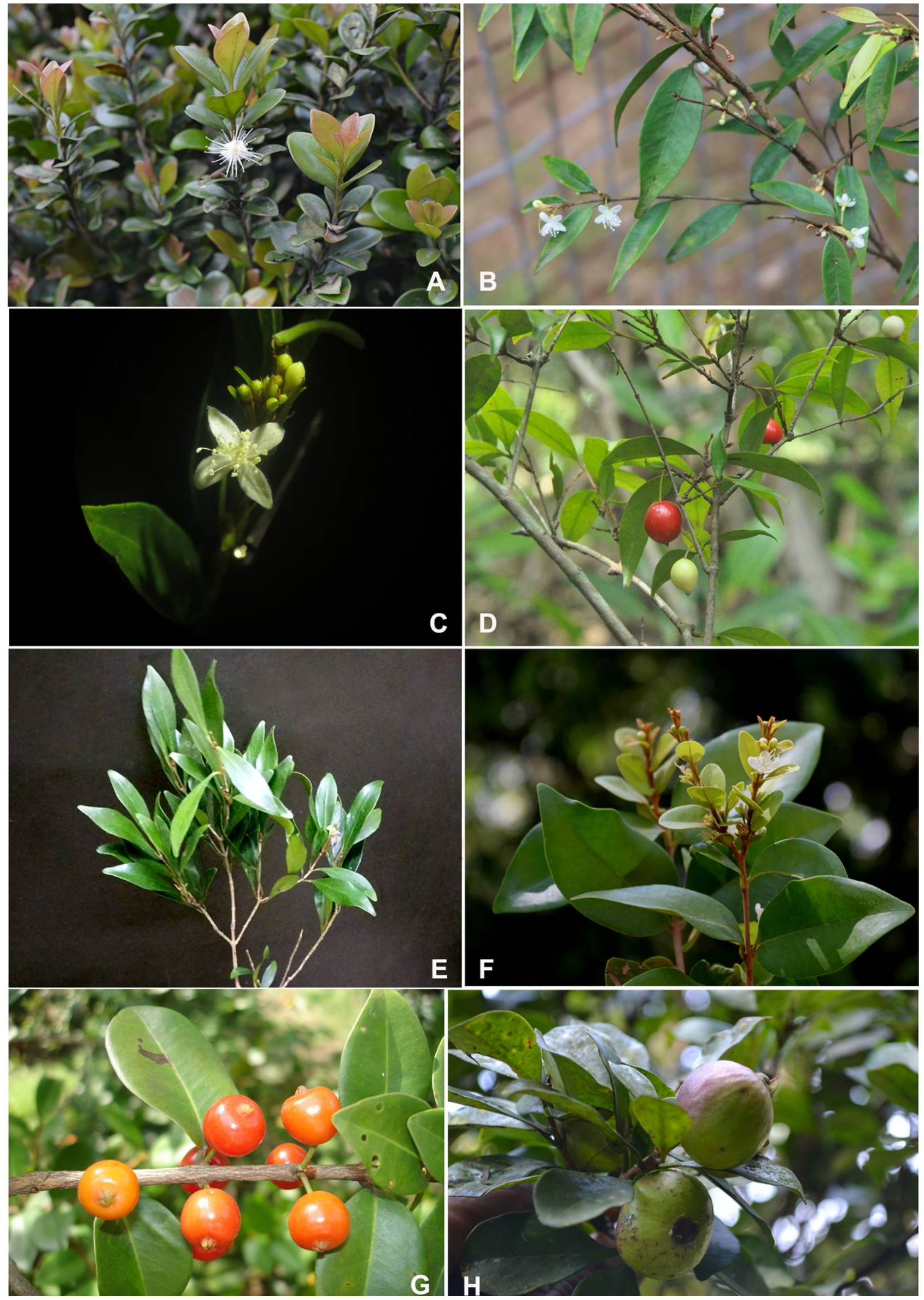

Fig. 2. A. Eugenia mabaeoides, B-D. E. mooniana, E. E. rottleriana, F - G. E. roxburghii, H. E. seithurensis.

obtusely acute at apex; lateral nerves $10-11$ pairs; petiole $0.6-1 \mathrm{~cm}$ long. Flowers $2 \mathrm{~cm}$ across, white, axillary or lateral, solitary $2-3$ fascicled; peduncle to
$2 \mathrm{~cm}$ long; pedicels $0.5-0.9 \mathrm{~cm}$ long, fulvous tomentose. Bracts small linear, rusty villous; bracteoles 2, lanceolate, c. 2 × $0.5 \mathrm{~mm}$, appresedly 
silky pubescent without. Calyx tube campanulate, 2-3 $\mathrm{mm}$ long; lobes 4, orbicular, 4-5 mm, tomentose without; petals orbicular, 8-10 mm, pinkish-white. Fruit globoid or ellipsoid, 2-2.5x $1.7-2.2 \mathrm{~cm}$, greenish-yellow, glabrous; seeds 1-2.

Type [Lectotype designated by Byng et al. (42)]: INDIA, Kerala, Travancore, near Chemunji, $1300 \mathrm{~m}$, April 1895, Bourdillon 580 (K) (Barcode K000821387)

Distribution: Tamil Nadu, Kerala; 750-1700 m.

Flowering \& Fruiting: September-February (Tamil Nadu); January-August (Kerala).

Eugenia floccosa Bedd., Fl. Sylv. S. India 1: t. 200. 1872.

Small trees, to $7 \mathrm{~m}$ high; young branchlets densely tawny- fulvous tomentose. Leaves broadly elliptic, 7.2-10.5 × 3.2-6 cm, thick coriaceous, cuneateattenuate at base, shortly acuminate with blunt tip to obtuse at apex, floccose beneath throughout when young or at least near base and on midrib when mature; lateral nerves, c. 12 pairs; petiole 1.5-2 cm long. Flowers large, c. $2.5 \mathrm{~cm}$ diam., white, solitary or in short few flowered terminal or axillary cymes to 2 cm long; pedicels $0.5-1.5 \mathrm{~cm}$ long. Bracts elliptic, 5-10 $\times 5 \mathrm{~mm}$, brown tomentose without; bracteole 2, large, obovate, c. $14 \times 11 \mathrm{~mm}$, brown wooly tomentose on either sides. Calyx tube broadly campanulate, to 10 $\mathrm{mm}$ long; lobes 4, broadly semi-lunar to ovateorbicular, c. $8 \mathrm{~mm}$, brown woolly on both side; petals 5, white, oblong, 5-8 $\times 4 \mathrm{~mm}$. Fruit globose or ellipsoid-pyriform, 2-4 $\times$ 2-3.4 cm, densely tomentose; seeds 2 .

Type: INDIA, Tamil Nadu, Tinnevelly Ghats, above Calcad, 3000- 4000 ft., R. Brown 199 (K) (Barcode K000821510).

Distribution: Tamil Nadu, Kerala, 1000-1500 m.

Flowering \& Fruiting: September-November (Tamil $\mathrm{Nadu}$; June-September (Kerala).

Eugenia indica (Wight) Chithra, Fl. Tamil Nadu 1: 153. 1983. Jossinia indica Wight, Icon. Pl. Ind. Orient. 2: t. 523. 1842. Eugenia jossinia Duthie, Hook. f., Fl. Brit. India 2: 500. 1879.

Large shrub to small trees, 5-7 m; branchlets densely rusty tomentose. Leaves elliptic-obovate or oblanceolate, $3-7 \times 1.5-3 \mathrm{~cm}$, thin coriaceous, acute or attenuate at base, obtuse at apex; lateral nerves 5-6 pairs; petiole 3-8 $\mathrm{mm}$ long, densely rusty tomentose. Flowers c. $1 \mathrm{~cm}$ across, axillary, solitary or paired, usually crowded at the end of branchlets; pedicels 1.5-2.5 cm long, densely rusty tomentose. Bracteoles 2, linear, c. $3 \mathrm{~mm}$ long. Calyx tube campanulate, 4-6 $\mathrm{mm}$ long; lobes 4, orbicular, equal, $5 \mathrm{~mm}$ across, fulvous tomentose; petals white, orbicular, 7-10 mm across, hairy. Fruit globose, 1-1.5 cm across, densely rusty tomentose; seeds few to many.

Type [Lectotype designated by Byng et al. (42)]: INDIA, Tamil Nadu, Shevagherry hills, near Courtallum, R.Wight, s.n. (K) (Barcode K000821508)
Distribution: Tamil Nadu, Kerala; 400-1200 m.

Flowering \& Fruiting: March-August.

Eugenia kalamii Shareef, E.S.S.Kumar, Shaju \& Prakashk, Pl. Sci. Today 5(4): 196. 2018. (Fig. 1. G-H).

Under-shrubs, to $1 \mathrm{~m}$ high; young shoots silvery pubescent. Leaves ovate-lanceolate, 2.5-9x 2.5-3.5 $\mathrm{cm}$, subcoriaceous, acute at base, narrowly acuminate at apex; lateral nerves 10-14 pairs; petioles 1.5-4 mm long. Flowers white, c. $1 \mathrm{~cm}$ across, solitary or paired, rarely 3-together, terminal, axillary or rarely lateral on a $3 \mathrm{~mm}$ long silvery pubescent peduncles; pedicels $1.5-6 \mathrm{~mm}$ long, silvery pubescent. Bracts 2, linear lanceolate, c.1.5x 0.7-1 mm long; bracteoles 2, ovate-deltoid, 0.8-1x $1 \mathrm{~mm}$, silvery pubescent. Calyx cup-shaped, to $2 \mathrm{x} 2 \mathrm{~mm}$; lobes 4, unequal, sub orbicular to elliptic-oblong, c.1.5x $1.2 \mathrm{~mm}$; petals 4, ovate-elliptic, 3.8-4.6x $3 \mathrm{~mm}$, white. Fruit succulent, depressed-globose, ellipsoid or rarely obovoid $11-14 \times 13-15 \mathrm{~mm}$, smooth, crimson on ripening; seed 1.

Type: INDIA, Kerala, Wayanad Distr., Periya, 840 m, 20.07.2016, S.M.Shareef 92402 (TBGT)

Distribution: Kerala.

Flowering \& Fruiting: July-September.

Eugenia mabaeoides Wight, Ill. Ind. Bot. 2: 131841. (Fig. 2. A).

Much branched shrub, 2-5 m high; young branchlets fugacious pubescent. Leaves spathulate to elliptic or obovate, $1.5-4 \times 0.5-2.5 \mathrm{~cm}$, coriaceous, narrowly attenuate at base, obtuse or rounded at apex; lateral nerves c.5-10 pairs; petiole 3-6 mm long. Flowers small, greenish-white with rosy tinge, 1-3, axillary; pedicels slender, $0.8-1 \mathrm{~cm}$ long, glabrous or slightly pubescent. Bracts 2, subulate, $1.5 \mathrm{~mm}$ long, fulvous tomentose. Bracteoles 2, linear, c.1.5 mm long, fulvous-tomentose. Calyx tube shallowly cup-shaped, $0.25-0.3 \mathrm{~cm}$ long; lobes 4 , broadly ovate, $0.28-0.4 \mathrm{~cm}$ long, glabrous or slightly pubescent without; Petals 4 , ovate-lanceolate, $0.5-0.6 \times 0.25-0.3 \mathrm{~cm}$, white. Fruit subglobose or ovoid, $0.5-0.6 \times 0.25-0.35 \mathrm{~cm}$, crimson or greenish purple.

Type [Lectotype designated by Byng et al. (42)]: SRI LANKA, Herb.Wight, s.n.(K) (Barcode K000821533)

Distribution: India (Tamil Nadu, Kerala), Sri Lanka; c.1500-1700 m.

Flowering \& Fruiting: March (Tamil Nadu); February-June (Kerala).

Note: Manickam et al. (28) reported Eugenia mabaeoides subsp. pedunculata (Trimen) P.S.Ashton from Tirunelveli hills as a new distributional record for India. Perusal of protologue and type specimen (Sri Lanka: Rangala range, September 1888, Trimen,s.n., K001000176-image!) provided by Trimen (46) is compared with the description and figure provided by Manickam et al. (28) showed remarkable differences. Hence the occurrence of $E$. mabaeoides subsp. pedunculata for India by Manickam et al. is a 

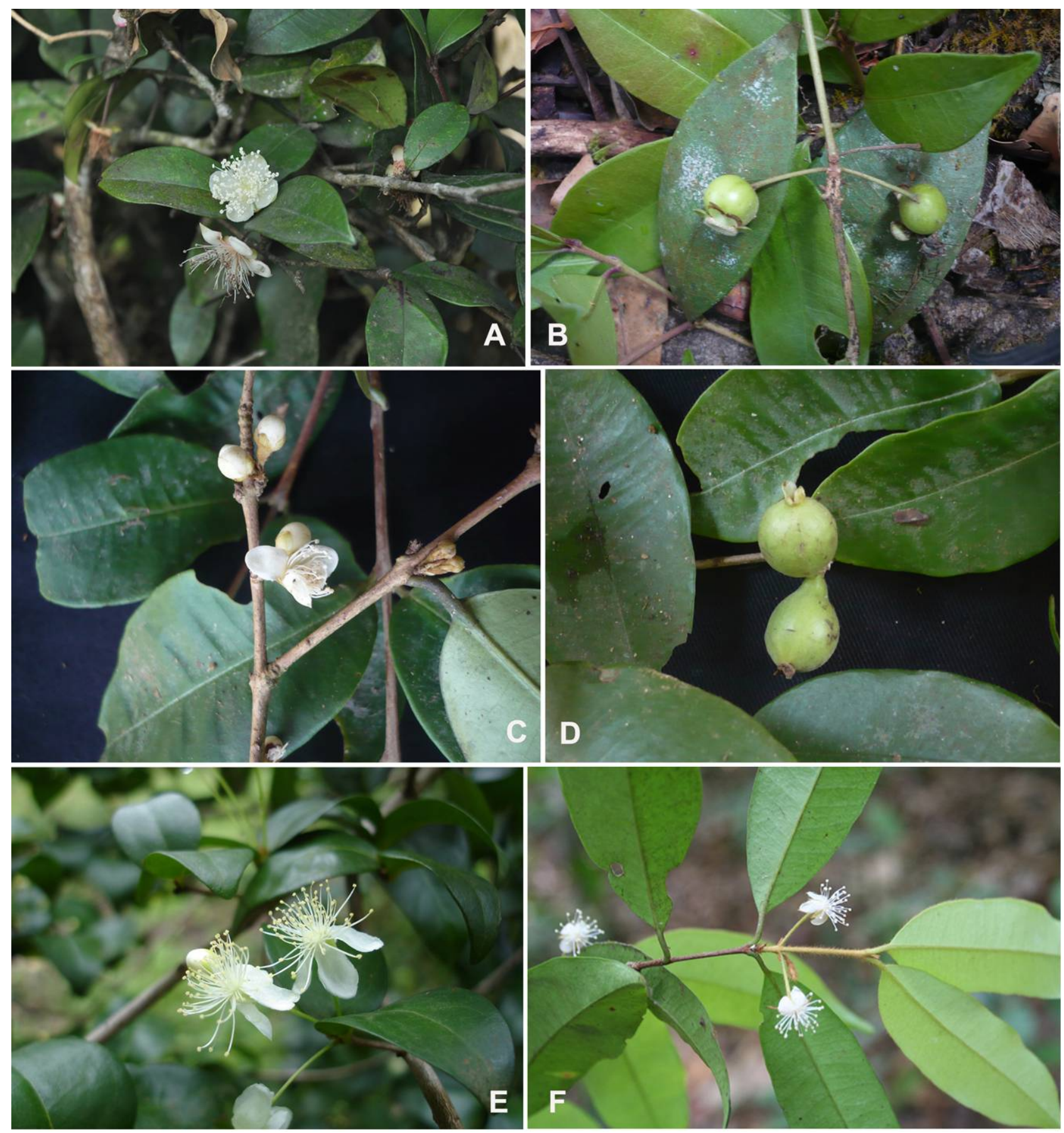

Fig. 3. A-B. Eugenia shettyana, C-D. E. terpnophylla var. keralensis, E. E. uniflora, F. E. wynadensis.

misidentification of a taxon, probably similar to $E$. manickamiana Murugan, pending further studies.

Eugenia macrosepala Duthie in Hook.f., Fl. Brit. India 2: 501. 1879.

Large shrubs to small trees, to $5 \mathrm{~m}$; branchlets pubescent; young flush reddish. Leaves elliptic to elliptic- oblong or oblong-lanceolate, 7-14.5x 2.7-5.5 $\mathrm{cm}$, chartaceous, acute at base, obtusely acuminate at apex; lateral nerves 10-15 pairs; petiole $0.5-0.8 \mathrm{~cm}$ long, pubescent when young. Flowers white, sessile, axillary or lateral from the leafless axils, solitary or in pairs. Bracteoles 2, linear, pilose, exceeding the calyx lobes. Calyx tube $6 \mathrm{~mm}$ long, clothed with long spreading white hairs without; lobes 4, narrowly lanceolate, ciliate, exceeding the petals; petals white, ovate-oblong, obtuse, ciliate. Fruit irregularly globose, $2.8 \mathrm{~cm}$ in diam., pubescent, deep orange when ripe; seeds 2-3.

Type: INDIA, Karnataka, N. Canara, J.E. Stocks, s.n., (K) (Barcode K000821512)

Distribution: Maharashtra, Goa, Karnataka; c.1000 m.

Flowering \& Fruiting: February-April (Maharashtra); January-April (Karnataka); January-August (Goa).

Note: Govaerts et al. (44) and POWO (45) treated Eugenia macrosepala Duthie as the synonym of Eugenia roxburghii DC., however, the former is very distinct by the large, 7-14.5 $\times 2.7-5.5 \mathrm{~cm}$, chartaceous leaves, (vs medium sized, 4-7 × 1-3 cm, coriaceous leaves); sessile flowers (vs pedicellate flowers; pedicel to $1.5 \mathrm{~cm}$ long), bracteoles exceeding calyx lobes (vs bracteoles not exceeding calyx lobes), narrowly 
lanceolate calyx lobes exceeding the petals (vs ovate calyx lobes, not exceeding the petals), ovate-oblong petals (vs elliptic petals) and narrowly lanceolate calyx lobes exceeding petals (vs ovate calyx lobes not exceeding petals), fruit irregularly globose, c. $2.8 \mathrm{~cm}$ diam., (vs subglobose, to $1.3 \mathrm{x} 1 \mathrm{~cm}$ diam.), seeds 1-2 (vs seeds 2-3) and hence it is reinstated as a distinct species here.

Eugenia manickamiana Murugan J. Econ. Taxon. Bot. 26(2): 414. 2002.

Small tree, to $4 \mathrm{~m}$ high; branchlets tawny-silvery pubescent. Leaves spathulate, 4-7x 1-3 cm, coriaceous, cuneate at base, broadly acuminate at apex; lateral nerves c. 8-15 pairs; petioles to $5 \mathrm{~mm}$ long. Inflorescence axillary or occasionally subterminal clusters; peduncle 1-2 mm long. Flowers white, 1-1.5 cm across, soliatary or rarely 3; pedicels 5-15 mm long. Bracteoles 1-2, linear-lanceolate, c. 1 $\mathrm{mm}$ long, pubescent. Calyx lobes 4, subequal, orbicular-ovate or concave, 2-3 mm across, puberulent without; petals 4, ovate-orbicular or concave, c. $7 \times 6 \mathrm{~mm}$, white.

Type: INDIA, Tamil Nadu, Tirunelveli Distr., Courtallum Hills, way to Attaikkadu from Therkkumalai Estate, 31 May 2001, Murugan 20932 (XCH).

Distribution: Tamil Nadu; 1200-1500 m.

Flowering: May-June.

Eugenia megamalayana Murugan \& Arum. in Taiwania 64(1): 23. 2019.

Trees, to $25 \mathrm{~m}$; branchlets fulvous tomentose. Leaves ovate-oblanceolate, $7-12.5 \times 3.5-4.5 \mathrm{~cm}$, chartaceous, rounded at base, acuminate at apex, fulvous tomentose when young; lateral nerves 8-12 pairs; petioles 1-1.2 cm long. Flowers c. $2.5 \mathrm{~cm}$ diam, white, solitary, axillary or rarely 3-flowered terminal racemes, to $2 \mathrm{~cm}$ long; peduncle $1-1.5 \mathrm{~cm}$ long. Bract and bracteoles linear, 4-7 $\mathrm{mm}$ long, fulvous tomentose. Calyx tube obconic; lobes 4, suequal, ovate, $0.5-0.7 \mathrm{~mm}$ diam.; petals 4 , orbicular, c. $1.2 \mathrm{~cm}$ diam. Drupes globose, c. $1.5 \mathrm{~cm}$ diam, fulvous tomentose; seeds 1 or 2.

Type: INDIA, Tamil Nadu, Theni Distr., Megamalai Wildlife Sanctuary, Vellimalai West Beat, on the way to Amman Estate, 17.07. 2017, 1422 m, C. Murugan \& S. Arumugam 134871 (CAL)

Distribution: Tamil Nadu; 1100-1500 m.

Flowering \& Fruiting: June-February.

Eugenia memecylifolia Talbot, J. Bombay Nat. Hist. Soc. 11: 236. 1897.

Large shrub or small tree. Leaves ovate, 3.8-5.8 $\times$ 2.5-3.8 cm, coriaceous, rounded at base, bluntly acuminate at apex; petiole $0.6-1 \mathrm{~cm}$ long. Flowers white, $1.2 \mathrm{~cm}$ across, sessile or subsessile, solitary or 2- many, fascicled in the leaf axils. Bracts 2, broadly ovate. Calyx lobes 4, ovate, tomentose without; petals
4, spreading. Fruit white, succulent, irregularly globose, 1.9-2.5 cm diam; seeds 1-2.

Type: INDIA, Karnataka, north Kanara, 3400 ft. (c. 1036 m.), 05 May 1894, Talbot 3127 (BSI)

Distribution: Karnataka

Flowering \& Fruiting: March-May.

Eugenia mooniana Wight, Ill. Ind. Bot. 2: 13. 1841. Eugenia thwaitesii Duthie, Fl. Brit. India 2: 506. 1879. Eugenia mooniana Gardner, London J. Bot. 2: 352. 1843. non Wight 1841; Eugenia neomooniana Sobral, Napaea 11: 36. 1995. (Fig. 2. B-D).

Shrub to small trees, $5 \mathrm{~m}$; branchlets puberulent. Leaves ovate-lanceolate to narrowly or broadly elliptic, very variable in size and shapes, 3-6 × 1.7-3.5 $\mathrm{cm}$, chartaceous, narrowly or broadly cuneate, shortly or to $2.5 \mathrm{~cm}$ long, attenuate acuminate at apex; lateral nerves c. 8-10 pairs; petiole 3-7 mm long. Flowers white, c. $1.5 \mathrm{~cm}$ across, solitary or paired or in lax terminal or axillary clusters; pedicels 1-2.5 cm long, very slender. Bracts 2, subulate, minute; bracteoles oblong, c. $2 \mathrm{~mm}$ long, fulvous tomentose. Calyx tube cup shaped, $2 \times 2 \mathrm{~mm}$; lobes 4 , oblong, 3-4 mm, slightly pubescent without; petals 4, elliptic-ovate, 5-10 × 2-5 mm, white. Fruit depressedglobose to ovoid, $1.3-1.5 \mathrm{~cm}$ diam, sparsely fulvous tomentose, crimson; seeds 1-2.

Type [Lectotype designated by Kumar et al. (38)]: INDIA, Tamil Nadu, Courtallum, Shevagherry hills, August 1836. Wight KD 1851 (K) (Barcode K000821519)

Distribution: India (Maharashtra, Karnataka, Tamil Nadu, Kerala), Sri Lanka; 0-1800 m.

Flowering \& Fruiting: October-February (Maharashtra); February-April (Karnataka); June-December (Tamil Nadu); October-July (Kerala).

Eugenia rottleriana Wight \& Arn., Prodr. Fl. Ind. Orient.: 331. 1834. (Fig. 2. E).

Much branched small trees, to $4 \mathrm{~m}$ high; branchlets tawny-fulvous tomentose. Leaves narrowlyoblanceolate, 3-7x 0.8-1.6 cm, coriaceous, attenuate at base, obtusely acuminate at apex; lateral nerves 5-8 pairs; petioles c. $5 \mathrm{~mm}$ long. Inflorescence axillary fascicled cymes or rarely solitary; peduncle to $5 \mathrm{~mm}$ long. Flowers white, c. $1 \mathrm{~cm}$ across; pedicels 1-2 cm long. Bract subulate, c. $1 \mathrm{~mm}$ long; bracteole linear, c. $2 \mathrm{~mm}$ long, pubescent. Calyx tube truncate, c. 2 x $2 \mathrm{~mm}$; lobes subequal, ovate, c. $2 \mathrm{~mm}$ long, fulvous tomentose; petals 4 or 5 , white, elliptic-ovate, c. 4-5x 2-3 mm. Fruits globose, c. $1 \mathrm{~cm}$ across, yellowish-green, rusty tomentose; seeds 1-2.

Type: INDIA, Peninsular India, R. wight 1075 (K) (Barcode K000821518)

Distribution: Tamil Nadu, Kerala; 400-1300 m.

Flowering \& Fruiting: March-September (Tamil Nadu); January-April (Kerala). 
Eugenia roxburghii DC., Prodr. 3: 271. 1828. Eugenia bracteata (Willd.) Raeusch. ex DC., Prodr. 3: 264. 1828. Eugenia bracteata var. fasciculata (Wall. ex Blume) Duthie, Fl. Brit. India 2: 502. 1879. Eugenia bracteata var. roxburghii (DC.) Duthie, Fl. Brit. India 2: 502. 1879. Eugenia fasciculata Wall. ex Blume, Mus. Bot. 1: 87. 1850. Eugenia heynei Rathakr. \& N.C.Nair, J. Econ. Taxon. Bot. 5: 232. 1984. Eugenia rothii Panigrahi in J. Econ. Taxon. Bot. 5: 994. 1984. Eugenia rothii var. fasciculata (Duthie) H.B.Naithani, Fl. Pl. India, Nepal \& Bhutan: 165. 1990; Eugenia willdenowii Wight, Ill. Ind. Bot. 2: 13. 1841. Eugenia zeylanica Roxb., Fl. Ind. ed.1832 2: 490. 1832. Myrtus bracteata Willd., Sp. Pl. 2: 969. 1799. Myrtus coromandelina J.König ex Roxb., Fl. Ind. ed.1832 2: 491. 1832. Myrtus heynei Spreng., Syst. Veg. 2: 482. 1825. Myrtus latifolia B.Heyne ex Roth, Nov. Pl. Sp. 232. 1821. Myrtus littoralis Roxb. ex Wight \& Arn., Prodr. Fl. Ind. Orient. 331. 1834. Myrtus quadripartita Royen ex Blume, Mus. Bot. 1: 87. 1850. Myrtus ruscifolia Willd., Sp. Pl. 2: 970. 1799. Syzygium bracteatum (Willd.) Raizada, Indian Forester 74: 336. 1948. Syzygium latifolium Blanco, Fl. Filip., ed. 2: 294. 1845. Syzygium ruscifolium (Willd.) Santapau \& Wagh, Bull. Bot. Surv. India 5: 109. 1964. (Fig. 2. F-G).

Much branched Shrubs or small trees, to $3 \mathrm{~m}$ high; branchlets tawny-pubescent. Leaves elliptic or lanceolate, 4-7x 1-3 cm, coriaceous acute at base, obtusely acuminate to caudate acuminate at apex; lateral nerves c. 10 pairs; petiole 6-9 $\mathrm{mm}$ long. Inflorescence axillary, 2-3 together or fascicled cymes or rarely solitary. Flowers white, c. $1.2 \mathrm{~cm}$ across; pedicels $0.6-1.5 \mathrm{~cm}$ long, tawny pubescent. Bracteoles 2, linear, $2.5 \mathrm{~mm}$ long. Calyx cup campanulate, 2.3-3 mm; lobes 4, ovate, c. 4x $3 \mathrm{~mm}$; petals white, elliptic, c. $6 \times 4 \mathrm{~mm}$. Fruits subglobose, to $1.3 \mathrm{x} 1 \mathrm{~cm}$ diam., green turn yellowish to orange or crimson, glabrous; seed 1-2.

Type: BANGLADESH, Sylhet, Roxburgh in Herb. Lambert in Herb. DC. (G; microfiche IDC), see also Wallich 3621-A (K; IDC microfiche 7394) and Roxburgh s.n., Icon Ined. 2502 (CAL, K)

Distribution: India (Assam, Meghalaya, West Bengal, Maharashtra, Goa, Karnataka, Tamil Nadu, Kerala), Sri Lanka, Bangladesh, Myanmar, Thailand, S. Vietnam; 100-900 m.

Flowering \& Fruiting: April-August (West Bengal); April-July (Maharashtra); April-August (Tamil Nadu); March-May (Kerala).

Eugenia seithurensis Gopalan \& S.R.Sriniv., J. Bombay Nat. Hist. Soc. 100: 78. 2003. (Fig. 2. H).

Trees, to $10 \mathrm{~m}$ high; branchlets glabrous. Leaves obovate, $3.2-5.4 \times 2.9-3.5 \mathrm{~cm}$, coriaceous, attenuate at base, abruptly acuminate at apex; petioles 3-7 mm long. Flowers axillary (solitary or in pairs) or in terminal 4-8 flowered umbels or cymes; pedicels 4-8 $\mathrm{mm}$ long. Bract 1, linear lanceolate or lanceolateelliptic, c. $5 \mathrm{~mm}$ long. Bracteoles 2, linear-lanceolate, c. $3 \mathrm{~mm}$ long. Calyx tube campanulate, 2-3 mm long; lobes 4 , subequal, broadly ovate, $2.5-3.5 \times 3-4 \mathrm{~mm}$, sparsely hairy; petals 4 , obovate or elliptic-oblong, c.
$11 \times 7 \mathrm{~mm}$. Fruit globose or subglobose, $1.7-3 \mathrm{~cm}$ across, greenish-yellow to pink; seeds 1-3.

Type: INDIA, Tamil Nadu, Virudhunagar Distr., Seithur Hills, between Deviar Estate and forest border of Kerala, 12 June 1979, c. 1350 m, S.R.Srinivasan 63532 (CAL).

Distribution: Tamil Nadu, Kerala; 1250-1580 m.

Flowering \& Fruiting: April-October (Tamil Nadu); May-January (Kerala).

Eugenia shettyana Murugan \& Gopalan, Nordic J. Bot. 23(5): 625. 2005. (Fig. 3. A-B).

Shrub to small trees, $4 \mathrm{~m}$ high; branchlets fulvous pubescent. Leaves elliptic-oblong, 3-7x 1.5-3 cm, chartaceous, rounded at base, abruptly acuminate at apex; lateral nerves $10-15$ pairs; petioles to $5 \mathrm{~mm}$ long. Flowers white, $2-3 \mathrm{~cm}$ across, supraaxillary, opposite; pedicels to $3.5 \mathrm{~cm}$ long. Bracteoles linear, c. $5 \mathrm{~mm}$ long. Calyx lobes subequal, 4-6 mm across, fulvous pubescent without; petals 4, white, ellipticoblong, c. 12x $8 \mathrm{~mm}$. Fruit globose, c. 1-2 cm across, fulvous pubescent; seeds 1-4.

Type: INDIA, Kerala, Idukki distr., Vaguvarai Shola (Top station), 1800-2000 m., 12 December 2003, C.Murugan 117638 (CAL)

Distribution: Tamil Nadu, Kerala; 1800-2150 m.

Flowering \& Fruiting: November-April.

Eugenia singampattiana Bedd., Icon. Pl. Ind. Or.: 65. 1874.

Small trees, to $5 \mathrm{~m}$ high; branchlets glabrous. Leaves ovate or elliptic-oblong, 5-12x 2.5-9 cm, chartaceous, cordate or rounded at base, obtuse or acuminate at apex; lateral nerves 8-10 pairs; petiole 3-10 mm long. Cymes terminal short racemes, to $2 \mathrm{~cm}$ long. Flowers white, c. $2.5 \mathrm{~mm}$ across; pedicels to $1 \mathrm{~cm}$ long. Bracts 2, linear-truncate; bracteoles elliptic-lanceolate, 0.8-1 $\mathrm{cm}$ long. Calyx tube $3 \mathrm{~mm}$ long; lobes 4, ovateorbicular, $4 \mathrm{~mm}$, slightly pubescent without; petals 4 white, orbicular-ovate, 12x $8 \mathrm{~mm}$ long. Fruits globose, $2-3 \mathrm{~cm}$ across, yellowish-orange to crimson red; seeds $1-4$.

Type [Lectotype designated by Byng et al. (42)]: INDIA, Tamil Nadu, Tinnevelly Hills, R.H. Beddome 2958 (BM) (Barcode BM000944115)

Distribution: Tamil Nadu, Kerala; 300-600 m.

Flowering \& Fruiting: February-August (Tamil Nadu); October-December (Kerala).

Eugenia terpnophylla Thwaites, Enum. Pl. Zeyl.: 114. 1859. (Fig. 3. C-D).

Medium tree, $2 \mathrm{~m}$ high; branchlets fulvous pubescent. Leaves elliptic-lanceolate, 6-11x 1.5-4.8 cm, thinly chartaceous, cuneate at base, acuminate at apex, to 2 $\mathrm{cm}$ long acumen; lateral nerves $6-10$ pairs; petiole to $10 \mathrm{~mm}$ long. Flowers white on short bracteate peduncles in axillary clusters; peduncle to $0.5 \mathrm{~mm}$ 
long; pedicel slender, to $5 \mathrm{~mm}$ long. Bracts and bracteoles linear, 1.5-2 mm long. Calyx campanulate, to $3 \times 2 \mathrm{~mm}$; lobes 4 , deltoid, acute at apex, $3 \times 2 \mathrm{~mm}$; petals 4, narrowly elliptic- oblong, 4x $2 \mathrm{~mm}$. Fruit globose, to $9 \mathrm{~mm}$ across; seeds 1-4.

Type: SRI LANKA, Kandy distr., Ambagamuwa, s.coll. 1860, Thwaites, G.H.K. CP2623 (BR) (Barcode BR0000005230167)

Distribution: India (Kerala), Sri Lanka; 20-1000 m.

Flowering \& Fruiting: March-October.

Eugenia terpnophylla var. keralensis Shareef, E.S.S.Kumar \& P.E.Roy, Nordic J. Bot. 29: 455. 2011. (Fig. 3. C-D).

Shrubs to small trees, to $6 \mathrm{~m}$; branchlets densely covered with golden brown hairs. Leaves narrowly or broadly elliptic, $6.5-14 \mathrm{x} \quad 2.4-4.5 \mathrm{~cm}$, thinly coriaceous, acute or obtuse at base, obtusely caudateacuminate at apex; lateral nerves 14-16 pairs; petioles 5-8 $\mathrm{mm}$ long. Flowers $1 \mathrm{~cm}$ across, white, 2-12 aggregated in axillary, terminal or lateral clusters or rarely on peduncle, to $1 \mathrm{~cm}$ long; pedicels to $3 \mathrm{~mm}$ long, pubescent. Bracts and bracteoles lanceolate, to $2 \mathrm{~mm}$ long. Calyx tube campanulate, to 4x $3 \mathrm{~mm}$, pubescent; lobes 4, suborbicular, c. $2 \times 1.7$ $\mathrm{mm}$, pubescent; petals 4, suborbicular, $5 \mathrm{x} 4.5 \mathrm{~mm}$, white. Fruit ellipsoid, c. 2.2x $1.9 \mathrm{~cm}$; seed 1, rarely 2.

Type: INDIA, Kerala, Pathanamthitta distr., Moozhiyar, 1100 m., 18 March 2010, S. M. Shareef \& P. E. Roy 69332 (TBGT)

Distribution: Kerala.

Flowering \& Fruiting: February-April.

Eugenia uniflora L., Sp. Pl. 470. 1753. Eugenia uniflora var. atropurpurea Mattos, Loefgrenia 85: 2. 1984. E. subcordata Wight \& Arn., Prodr. Fl. Ind. Orient. 331. 1834. (Fig. 3. E).

Shrub to small trees, to $8 \mathrm{~m}$; branchlets glabrous. Leaves ovate or oblong-elliptic, $3.6-5 \times 1.3-3 \mathrm{~cm}$, chartaceous, acute or obtuse at base, acute at apex; lateral nerves 5-6 pairs; petiole 2-3mm long. Flowers white, -3 , axillary; peduncle to $2 \mathrm{~cm}$ long. Bracts 3-5 $\mathrm{mm}$ long; bracteoles linear, 1-2 $\mathrm{mm}$ long. Calyx tube funnel shaped, $2 \times 2 \mathrm{~mm}$; lobes 4 , oblong to deltoid, to $4 \mathrm{~mm}$; petals 4 , orbicular to obovate, 7-8 $\mathrm{mm}$, white. Fruits globose, $1.5 \times 1.5-3 \mathrm{~cm}$, prominently ribbed, succulent, green turns yellowish to crimson; seeds 1 or 2 .

Type [Lectotype designated by McVaugh (43)]: SRI LANKA, Micheli, Nov. Pl. Gen. t. 108. 1729.

Distribution: Native to Brazil, Cultivated in India; 100-600 m.

Flowering \& Fruiting: September-November.

Eugenia velliangiriana Murug., V.Ravich., Murugan \& Arum.,Webbia 74(1): 23. 2019.
Medium-sized or large trees, to $18 \mathrm{~m}$ high; branchlets sparsely pubescent. Leaves obovate-lanceolate, 2.5-4x 1-1.7 cm, coriaceous, cuneate at base, shortly acuminate at apex; lateral veins fainted; petiole 2-3 $\mathrm{mm}$ long. Flowers $0.5-0.8 \mathrm{~cm}$ across, white, solitary, axillary, binate with opposite axils; pedicel $0.5-1 \mathrm{~cm}$ long. Bracts 2, linear-lanceolate, c. $3 \mathrm{~mm}$ long; bracteoles ovate, c. $3 \mathrm{~mm}$ long. Calyx tube sparsely pubescent; lobes 4, unequal, oblong, 2-4.2x 2.2-2.5 $\mathrm{mm}$, sparsely pubescent; petals 4 , orbicular, c. $0.7 \mathrm{~cm}$ long, clawed at base, Drupes oblongoid-ellipsoid, c. $1.5 \mathrm{~cm}$ across, sparsely pubescent; seed 1.

Type: INDIA, Tamil Nadu, Coimbatore Distr., Velliangiri hills, 1359 m, 12 December 2018, M. Murugesan \& V. Ravichandran 139190 (MH).

Distribution: Tamil Nadu; 1359-1700 m.

Flowering \& Fruiting: October-December.

Eugenia wynadensis Bedd. in Madras J. Lit. Sci. III, 1: 47.1864. Meteoromyrtus wynadensis (Bedd.) Gamble in Bull. Misc. Inform. Kew 1918: 241.1918. (Fig. 3. F).

Small trees, to $6 \mathrm{~m}$ high; branchlets fulvous tomentose. Leaves elliptic, elliptic-oblong or oblonglanceolate, 4-9x 1.5-3.5 cm, chartaceous, cuneate at base, caudate-acuminate at apex; lateral nerves 6-10 pairs; petioles 5-10 mm long. Inflorescence axillary or terminal racemes, to $1 \mathrm{~cm}$ long; peduncle c. $5 \mathrm{~mm}$ long. Flowers, $1.5 \mathrm{~cm}$ across, white, solitary or in pairs; pedicels slender, 1-1.5 cm long, rusty tomentose. Bracteoles linear, c. $2 \mathrm{~mm}$ long, fulvous tomentose. Calyx lobes 4, lanceolate, c. 3x $2 \mathrm{~mm}$, subequal, fulvous tomentose; petals 4 , white, ellipticovate, c. 10x $5 \mathrm{~mm}$. Fruits globose or pot shaped, c. 2x $2 \mathrm{~cm}$, fleshy when mature, greenish, sparsely fulvous hairy; seeds 1-4.

Type [Lectotype designated by Byng et al. (42)]: INDIA, Kerala, Wynad (Devallikottah), Beddome 2943 (K) (Barcode K000821504)

Distribution: Karnataka, Tamil Nadu, Kerala; 650-1200 m.

Flowering \& Fruiting: September-January (Karnataka); February-August (Tamil Nadu \& Kerala).

\section{Acknowledgements}

The authors are thankful to the Director, JNTBGRI for encouragement and support.

\section{Authors' contributions}

SSM drafted the manuscript. ESSK has checked the nomenclature part and modified the manuscript. Both authors read and approved the final manuscript.

\section{Conflict of interest}

The authors declare that they have no competing interests. 


\section{References}

1. WCSP. World Checklist of Selected Plant Families [Internet]. Facilitated by the Royal Botanic Gardens, Kew [cited 2020 February 22]. Available from: http://wcsp.science.kew.org

2. Mazine FF, Bünger MO, Faria JEQ, Lucas E, Souza VC. Sections in Eugenia (Myrteae, Myrtaceae): nomenclatural notes and a key. Phytotaxa. 2016;289(3):225-36. https://doi.org/10.11646/ phytotaxa.289.3.2

3. Giaretta A, Marcelo DCS, Luis Fernando TDM, Ariane LP. Two new species of Eugenia (Myrtaceae) from the Atlantic forest of Espirito Santo, Brazil. Phytotaxa, 2018;336(2):181-89. https:// doi.org/10.11646/phytotaxa.336.2.5

4. Duthie JF. Myrtaceae. In: Hooker JD, editor. Flora of British India Vol. 2, Reeve and Co., London; 1879. p. 470-506

5. Ingle HD, Dadswell HE. The anatomy of the timbers of the south-west Pacific area. Australian Journal of Botany. 1953;1:353-401. https://doi.org/10.1071/BT9530353

6. Schmid R. A resolution of the Eugenia-Syzygium controversy (Myrtaceae). American Journal of Botany. 1972;59:423-36. https://doi.org/10.1002/j.1537-2197.1972.tb10113.x

7. Schmid R. Floral anatomy of Myrtaceae. I. Syzygium. Botanische Jahrbücher fur Systematik, Pflanzengeschichte und Pflanzengeographie., 1972; 92:435-89

8. Schmid R. Floral anatomy of Myrtaceae. II. Eugenia. Journal Arnold Arboretum. 1972; 53:336-63

9. Pike KM. Pollen morphology of Myrtaceae from the south-west Pacific area. Australian Journal of Botany. 1956;4:13-53. https:// doi.org/10.1071/BT9560013

10. Wilson PG, O’Brien MM, Heslewood MM, Quinn CJ. Relationships within Myrtaceae sensu lato based on a matK phylogeny. Plant Systematic Evolution 2005;251:3-19. https://doi.org/ 10.1007/s00606-004-0162-y

11. Cooke T. Myrtaceae. In: Cooke T, editor. The Flora of the Presidency of Bombay. Vol. 1. Taylor \& Francis, London; 1903. p. 48697. https://doi.org/10.5962/bhl.title.10884

12. Talbot WA. Myrtaceae. In: Talbot WA, editor. Forest Flora of the Bombay Presidency and Sind. Vol. 1. Government of Poona, India; 1911. p. 29-49

13. Gamble JS. Myrtaceae. In: Gamble JS, editor. Flora of the Presidency of Madras. Vol. 1. Adlard \& Sons Ltd., London; 1919. p. 470-86

14. Kanjilal UN, Kanjilal PC, Das A. Myrtaceae. In: Kanjilal UN, Kanjilal PC, Das A, editors. Flora of Assam. Vol. 2. Government of Assam, Shillong, India; 1938. p. 257-87

15. Prain D. Myrtaceae. In: Prain D, editor. Bengal Plants. Vol. 1. Bishen Singh Mahendra Paul Singh, Dehra Dun; 1981. p. 485-92

16. Gandhi KN. Myrtaceae. In: Saldanha CJ, Nicolson DH, editors. Flora of Hassan District, Karnataka, India. Amerind publishing Co. Pvt. Ldt., New Delhi; 1976. p. 273-83

17. Deb DB. Myrtaceae. In: Deb DB, editor. The Flora of Tripura State. Vol. 1. Today \& Tomorrow's Printers and Publishers, India; 1981. p. $366-73$

18. Chithra V. Myrtaceae. In: Nair NC, Henry AN, editors. Flora of Tamil Nadu, India. Series 1: Analysis. Vol. 1. Botanical Survey of India, Coimbatore; 1983 . p. 150-58

19. Haridasan K, Rao RR. Myrtaceae. In: Haridasan K, Rao RR, editors. Forest Flora of Meghalaya. Vol. 1, BSMPS, Dehradun, India; 1985. p. 386-403

20. Saldanha CJ. Myrtaceae. In: Saldanha CJ, editor. Flora of Karnataka. Vol. 2. Oxford \& IBH Publishing, New Delhi, India; 1996. p. 23-32

21. Pullaiah T, Chennaiah E. Myrtaceae. In: Pullaiah T, Chennaiah E, editors. Flora of Andhra Pradesh. Vol. 1. Scientific Publishers, Jodhpur, India; 1997. p. 381-85

22. Almeida MR. Myrtaceae. In: Almeida MR, editor. Flora of Maharashtra. Vol. 2. Orient Press, Mumbai; 1998. p. 233-38

23. Kulkarni BG. Myrtaceae. In: Singh NP, Lakshminarasimhan P, Karthikeyan S, Prasanna PV, editors. Flora of Maharashtra State. Vol. 2. Botanical Survey of India, Howrah, India; 2001. p. 7-16

24. Mohanan N, Sivadasan M. Myrtaceae. In: Mohanan N, Sivadasan M, editors. Flora of Agasthyamala. Vedams Books International, India; 2002. p. 252-66
25. Naithani HB, Sahni KC, Bennet SSR. Myrtaceae. In: Naithani HB, Sahni KC, Bennet SSR, editors. Forest Flora of Goa. International Book Distributors, Dehra Dun; 1997. p. 282-89

26. Nayar TS, Rasiya Beegam A, Mohanan N, Rajkumar G. Myrtaceae. In: $\quad$ Nayar TS, Rasiya Beegam A, Mohanan N, Rajkumar G, editors. Flowering Plants of Kerala: A Handbook. Tropical Botanic Garden and Research Institute, Thiruvananthapuram, India; 2006. p. 445-54

27. Nayar TS, Rasiya Beegam A, Sibi M. Myrtaceae. In: Nayar TS, Rasiya Beegam A, Sibi M, editors. Flowering Plants of the Western Ghats, India Vol. 1. Dicots. Jawaharlal Nehru Tropical Botanical Garden and Research Institute; 2014. p. 669-87

28. Manickam VS, Murugan C, Jothi GJ. Myrtaceae. In: Manickam VS, Murugan C, Jothi GJ, editors. Flora of Tirunelveli Hills (Southern Western Ghats) - Vol. 1 - Polypetalae. Bishen Singh Mahendra Pal Singh, Dehradun; 2008. p. 387-95

29. Chora C. Myrtaceae. In: Paul YK, Lakshminarasimhan P, Chow dhery HJ, Dash SS, Singh P, editors. Flora of West Bengal. Vol. 2. Botanical Survey of India, Kolkotta; 2015. p. 318-32

30. Rathakrishnan NC. Myrtaceae. In: Murthy GVS, Nair VJ, editors. The Flora of Kerala. Vol. 2, Botanical Survey of India, Kolkata; 2016. p. 349-72

31. Murugan C. New species of Xanthophyllum Roxb.(Xanthophyllaceae) and Eugenia L. (Myrtaceae) from Peninsular India. Journal of Economic and Taxonomic Botany. 2002;26:413-18

32. Gopalan R, Srinivasan SR. A new species of Eugenia L. (Myrtaceae) from Seithur Hills, Tamil Nadu, India. Journal of Bombay Natural History Society. 2003;100:78-80

33. Kumar ESS, Yeragi SS. Eugenia terpnophylla Thw. (Myrtaceae): a new record for India. Rheedea. 2003;13:39-41

34. Murugan C, Gopalan R. A new species of Eugenia (Myrtaceae) from the Western Ghats, India. Nordic Journal of Botany. 2005 23:625-27. https://doi.org/10.1111/j.1756-1051.2003.tb00443.x

35. Gopalan R, Murugan C. Eugenia agasthiyamalayana (Myrtaceae), a new species from the southern Western Ghats of India. Indian Journal of Forestry. 2008;31:641-42

36. Shareef SM, Kumar ESS, Roy PE. Eugenia terpnophylla var. ker alensis (Myrtaceae), a new variety from Kerala, India. Nordic Journal of Botany. 2011;29:455-57. https://doi.org/10.1111/ j.1756-1051.2011.01126.x

37. Shareef SM, Kumar ESS, Shaju T, Prakashkumar R. Eugenia kalamii (Myrtaceae), a new species from Western Ghats, India. Plant Science Today. 2018;5(4):196-200. https://doi.org/10.14719/ pst.2018.5.4.429

38. Kumar ESS, Veldkamp JF, Shareef SM. Notes on Eugenia gracilis, Eugenia mooniana and Eugenia phillyreoides (Myrtaceae) Webbia. 2014;69(1):101-03. http://dx.doi.org/ 10.1080/00837792.2014.895890

39. Murugan C, Arumugam S. Eugenia megamalayana sp. nov (Myrtaceae) - A new species from the Western Ghats, India. Taiwania. 2019;64(1):4-8

40. Murugesan M, Ravichandran V, Murugan C, Arumugam S. Eugenia velliangiriana (Myrtaceae), a new species from the Western Ghats, India. Webbia. 2019;74(1):23-27. https://doi.org/ $10.1080 / 00837792.2019 .1605234$

41. Kumar ESS, Shareef SM, Roy PE. Rediscovery and lectotypification of Eugenia argentea Bedd. (Myrtaceae) - An endemic and endangered species of India. Journal of Scientific Research 2011;3(1):213-16. https://doi.org/10.3329/jsr.v3i1.6210

42. Byng JW, Wilson PG, Snow N. Typifications and nomenclatural notes on Indian Myrtaceae. Phytotaxa. 2015;217(2):101-16. https://doi.org/10.11646/phytotaxa.217.2.1

43. McVaugh R. Nomenclatural notes on Myrtaceae and related families. Taxon. 1956;5(6):133-47. https://doi.org/10.2307/1217674

44. Govaerts R, Sobral N, Ashton P, Barrie F, Holst BK, Landrum LL, et al. World Checklist of Myrtaceae. Kew Publishing, Royal Botanic Gardens, Kew; 2008. p. 1-455

45. POWO. Plants of the World Online [Internet]. Facilitated by the Royal Botanic Gardens, Kew [cited 2020 March 01]. Available from: http://www.plantsoftheworldonline.org

46. Trimen, H. Additions to the flora of Ceylon 1885-88. Journal of Botany. 1889;27:162 